\title{
On The Stability of Conditional Homomorphisms in Lie $C^{*}$-algebras
}

\section{Eshaghi $\mathbf{M}^{1,2}$, Abbaszadeh $\mathbf{S}^{1,3 *}$ and Manuel De la Sen ${ }^{4}$}

${ }^{1}$ Department of Mathematics, Faculty of Mathematics, Statistics and Computer Sciences, Semnan University, Semnan 35195-363, Iran

${ }^{2}$ Center of Excellence in Nonlinear Analysis and Applications, Semnan University, Semnan, Iran

${ }^{3}$ Young Researchers and Elite Club, Malayer Branch, Azad University, Malayer, Iran

${ }^{4}$ Department of Electricity and Electronics, University of the Basque Country, Spain

\begin{abstract}
During the last years several papers studying conditional functional equations have appeared. They mostly deal with equations satisfied on some restricted domain and many among them concern equations postulated for orthogonal vectors. In this paper, we define the conditional homomorphisms with the predecessor defined by $Y$ $(x)=\gamma(y)$ with an even mapping $\mathrm{Y}$. Then, using a fixed point theorem, we investigate the stability of the conditional homomorphisms in Lie $C^{*}$-algebras.
\end{abstract}

Keywords: Conditional homomorphism; Contractively sub homogeneous; Expansively super homogeneous; Fixed point theorem; Stability

\section{Introduction}

The problem of solving equations on spheres is an important part of computational aspects, ordinary differential equations and partial differential equations [1-3]. The development of computational methods for solving partial differential equations on the sphere is complicated by problems that result from the spherical coordinate system itself. However, some of the numerical methods for solving vector differential equations are applicable to any vector differential equation on the sphere.

The functional equation $(\xi)$ is stable if any function $f$ satisfying the equation $(\xi)$ approximately is near to the true solution of $(\xi)$. An interested reader can find more information on such problems with the emphasis on functional equations in [4-8].

Let $X$ and $Y$ are two Banach spaces. Consider $f: X \rightarrow Y$ to be a mapping such that $f(t x)$ is continuous in $t \in \mathbb{R}$ for each fixed $x \in X$. Assume that there exist constants $\varepsilon \geq 0$ and $p \in[0,1)$ such that

$$
\|f(x+y)-f(x)-f(y)\| \leq \varepsilon\left(\|x\|^{p}+\|y\|^{p}\right)
$$

For all $x, y \in X$, Rassias [6] showed that there exists a unique $\mathbb{R}$ -linear mapping $T: X \rightarrow Y$ such that

$$
\|f(x)-T(x)\| \leq \frac{2 \varepsilon}{2-2^{p}}\|x\|^{p}
$$

For all $x \in X$, A generalization of the theorem of Rassias was obtained by Gavruta [9] by replacing the unbounded Cauchy difference by a general control function $\varphi: G \times G \rightarrow[0, \infty)$ in the spirit of Rassias approach.

In 1994, Alsina and Garcia-Roig [10] solved the conditional equation

$$
f(x+y)=f(x)+f(y)
$$

whenever $\|\mathrm{x}\|=\|\mathrm{y}\|$ for continuous mapping $\mathrm{f}$ from a real inner product space $(X,(. \mid)$.$) with \operatorname{dim} X \geq 2$ into a real topological linear space $Y$. They recognized the connection between this equation and the orthogonally additive functional equation. They also obtained the linearity of such a function $\mathrm{f}$ in the case where $Y=R^{n}$.

Sikorska [11] studied a generalized stability of Cauchy and Jensen functional equations, where the respective Cauchy or Jensen differences are approximated by arbitrary functions. Moreover, [12] Sikorska solved the conditional Pexider functional equation on prescribed sets being generalizations of spheres. We refer the reader to [13-17] for some interesting results on the stability of conditional functional equations.

In this paper, we apply a fixed point theorem to prove the stability of conditional homomorphisms using contractively subhomogeneous and expansively superhomogeneous functions.

The paper is organized as follows. In Section 2, some necessary preliminaries and summarization of some previous known results are presented. The concepts of conditional homomorphism and conditional Jordan homomorphism in Lie $C^{*}$-algebras are introduced. In Section 3, we deal with the stability of conditional homomorphisms and conditional Jordan homomorphisms in Lie $C^{*}$-algebras. Finally, a conclusion is given in Section 4.

\section{Preliminaries}

Ger and Sikorska [18] solved the conditional functional equation (1.1) with the norm replaced by an abstract function fulfilling suitable conditions.

Theorem 2.1: [18] Let $X$ be a real linear space with $\operatorname{dim} X \geq 2$, ( $Y$, + ) be an abelian group, $Z$ be a given nonempty set and let $\gamma: X \rightarrow Z$ be an even mapping $\gamma(-x)=\gamma(x), x \in X$ such that

(c) for any two linearly independent vectors $x, y \in X$ there exist linearly independent vectors $u, v \in \operatorname{Lin}\{x, y\}$ such that $\gamma(u+v)=\gamma(u-v)$,

$\left(c_{2}\right)$ if $x, y \in X, \gamma(x+y)=\gamma(x-y)$, then $\gamma(\alpha x+y)=\gamma(\alpha x-y)$ for all $\alpha \in \mathbb{R}$,

$\left(\mathrm{c}_{3}\right)$ for all $x \in X$ and $\lambda \in \mathbb{R}_{+}$there exists a $y \in X$ such that $\gamma(x+y)=\gamma$ $(x-y)$ and $\gamma((\lambda+1) x)=\gamma((\lambda-1) x-2 y)$.

${ }^{*}$ Corresponding author: Abbaszadeh S, Department of Mathematics, Faculty of Mathematics, Statistics and Computer Sciences, Semnan University, Iran, Tel: 982333366296; E-mail: abbaszadeh@semnan.ac.ir

Received March 24, 2015; Accepted June 27, 2015; Published June 30, 2015

Citation: Eshaghi M, Abbaszadeh S, Sen MD (2015) On The Stability of Conditional Homomorphisms in Lie $C^{*}$-algebras. J Generalized Lie Theory Appl 9: 220. doi: 10.4172/1736-4337.1000220

Copyright: @ 2015 Eshaghi M, et al. This is an open-access article distributed under the terms of the Creative Commons Attribution License, which permits unrestricted use, distribution, and reproduction in any medium, provided the original author and source are credited. 
If $f: X \rightarrow Y$ satisfies the condition

$\gamma(x)=\gamma(y)$ implies $f(x+y)=f(x)+f(y), \quad x, y \in X$,

Then $f$ is additive.

They dealt also with the Hyers-Ulam stability problem cf. [4,5] for such a more general version of (1.1). In order to make the above assumptions more readable, they gave the following example.

Example 2.2: Let $(X,(. \mid)$.$) be a real inner product space with \operatorname{dim} X$ $\geq 2, Z \in \mathbb{R}$ and $\gamma(x)=\|x\|, x \in X$. Then function $\gamma$ satisfies $\left(c_{1}\right)-\left(c_{3}\right)$.

A $C^{\star}$-algebra A endowed with the Lie product $[x, y]=x y-y x$ on $A$ is called a Lie $C^{*}$-algebra. The stability problems of functional equations between $C^{*}$-algebras have been investigated by a number of authors [19-22].

Definition 2.3: A $\mathbb{C}$-linear mapping $H$ of a Lie $C^{\star}$-algebra $A$ to a Lie $C^{*}$-algebra $B$ is called a conditional homomorphism if

$$
\mathrm{H}([x, y])=[\mathrm{H}(x), \mathrm{H}(y)]
$$

Holds for all $x, y \in A$ with $\gamma(x)=\gamma(y)$.

Definition 2.4: A $\mathbb{C}$-linear mapping $H: A \rightarrow B$ is said to be a conditional Jordan homomorphism if

$H([x, y]+[y, x])=[H(x), H(y)]+[H(y), H(x)]$

For all $x, y \in A$ with $\gamma(x)=\gamma(y)$.

For explicitly later use, we state the following theorem.

Theorem 2.5: (Banach) Let $(X, d)$ be a complete metric space and consider a mapping $\Lambda: X \rightarrow X$ as a strictly contractive mapping, that is

$$
d(\Lambda x, \Lambda y) \leq L d(x, y)
$$

For all $x, y \in X$ and for some (Lipschitz constant) $0<L<1$. Then there exists a unique $a \in X$ such that $\Lambda a=a$. Moreover, for each $x \in X$,

$$
\lim _{n \rightarrow \infty} \Lambda^{n} x=a
$$

and in fact for each $x \in X$,

$$
d(x, a) \leq \frac{1}{1-L} d(x, \Lambda x) \text {. }
$$

Let $A, B$ be real vector spaces. We recall that if there exists a constant $L$ with $0<L<1$ such that a $n$-times mapping $\rho: A^{n}=\overbrace{A \times \cdots \times A}^{n \text {-times }} \rightarrow B$ satisfies

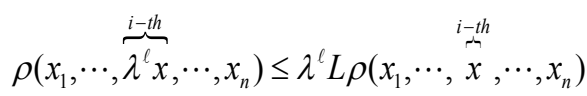

For all $x, x_{j} \in A(1 \leq j \neq i \leq n)$ and all positive integers $\lambda$, then we say that $\rho$ is $n$-contractively sub homogeneous if $\ell=-1$, and $\rho$ is $n$-expansively superhomogeneous if $l=-1$. It follows by the above inequality that $\rho$ satisfies the following properties:

$$
\begin{aligned}
& \rho(x_{1}, \cdots, \overbrace{\lambda^{\ell k}}^{i-t h} x, \cdots, x_{n}) \leq\left(\lambda^{\ell} L\right)^{k} \rho\left(x_{1}, \cdots, \stackrel{i-t h}{\stackrel{x}{x}}, \cdots, x_{n}\right), k \in \mathbb{N}, \\
& \rho\left(\lambda^{\ell} x, \cdots, \lambda^{\ell} x\right) \leq\left(\lambda^{\ell} L\right)^{n} \rho(x, \cdots, x)
\end{aligned}
$$

For all $x, x_{j} \in A(1 \leq j \neq i \leq n)$ and all positive integers $\lambda$.

Remark 2.6: If $\rho$ is $n$-contractively sub-additive then $\rho$ is contractively sub homogeneous of degree $n$ and if $\rho$ is $n$-expansively super-additive, then $\rho$ is and expansively super homogeneous of degree $n$.

\section{The Main Results}

Throughout this section, let $A$ and $B$ be Lie $C^{*}$-algebras, $\operatorname{dim} A \geq$ 2 and $\gamma$ be an even mapping from $A$ to a nonempty set $Z$, satisfying the conditions $\left(\mathrm{c}_{1}\right)-\left(\mathrm{c}_{3}\right)$ and for all $x, y \in A, \gamma(x)=\gamma(y)$ implies that $\gamma\left(2^{\ell} x\right)=\gamma\left(2^{\ell} y\right)$ where $\ell \in\{-1,1\}$. Let $\mathbb{T}_{1 / n_{0}}^{1}:=\left\{e^{i \theta} ; 0<\theta<2 \pi / n_{0}\right\}$.

Let $\varphi: \mathcal{A}^{3} \rightarrow(0, \infty)$ be either 3 -expansively super homogeneous mappings for $\ell=-1$ or 3 -contractively sub homogeneous mappings for $\ell=1$, with constant $0<L=L(\ell)<\frac{1}{\sqrt{2}}$. Let $\phi: \mathcal{A}^{2} \rightarrow(0, \infty)$ be either 2expansively super homogeneous mappings for $\ell=-1$ or 2 -contractively sub homogeneous mappings for $\ell=1$, with constant $0<L=L(\ell)<1$.

Lemma 3.1: Let $\quad \mathcal{A} \rightarrow \mathcal{B}$ be an additive function such that $f$ $(t x)=t f(x)$ for all $t \in \mathbb{T}_{1 / n_{0}}^{1}$ and $x \in A$. Then the function $f$ is $\mathbb{C}$-linear [23].

Theorem 3.2: Suppose that $f_{i}: \mathcal{A} \rightarrow \mathcal{B}(i=1,2,3)$ are mappings fulfilling

$$
\begin{aligned}
& \left\|f_{1}(x+y)-f_{2}(x)-f_{3}(y)+f_{i}(t z)-t f_{i}(z)\right\| \leq \varphi(x, y, z), \\
& \left\|f_{1}([x, y])-\left[f_{2}(x), f_{3}(y)\right]\right\| \leq \phi(x, y)
\end{aligned}
$$

For all $t \in \mathbb{T}_{1 / n_{0}}^{1}$ and $x, y, z \in A$ with $\gamma(x)=\gamma(y)$. If $f_{3}$ is an odd mapping and $f_{i}(0)=0(i=1,2,3)$, then there exists a unique conditional homomorphism $H: \mathcal{A} \rightarrow \mathcal{B}$ such that

$$
\begin{aligned}
& \left\|f_{1}(x)-H(x)\right\| \leq \frac{L^{1-\ell}}{2(1-L)} \psi\left(x, y_{0}\right), \\
& \left\|f_{2}(x)-H(x)\right\| \leq \frac{L^{3-\ell}}{1-L} \psi\left(x, y_{0}\right)+\frac{1}{2}(\varphi(x, x, 0)+\varphi(x,-x, 0)), \\
& \left\|f_{3}(x)-H(x)\right\| \leq \frac{L^{3-\ell}}{1-L} \psi\left(x, y_{0}\right)+\frac{1}{2}(\varphi(x, x, 0)+\varphi(x,-x, 0)),
\end{aligned}
$$

Where the mapping $\psi: \mathcal{A}^{2} \rightarrow(0, \infty)$ is defined by

$$
\begin{aligned}
\psi\left(x, y_{0}\right) & =\varphi(x, x, 0)+\varphi\left(y_{0}, x, 0\right)+\varphi\left(x,-y_{0}, 0\right)+\varphi\left(y_{0},-y_{0}, 0\right) \\
& +\varphi\left(\frac{x+y_{0}}{2}, \frac{x+y_{0}}{2}, 0\right)+\varphi\left(\frac{x-y_{0}}{2}, \frac{x-y_{0}}{2}, 0\right) \\
& +\varphi\left(\frac{x+y_{0}}{2}, \frac{x-y_{0}}{2}, 0\right)+\varphi\left(\frac{x-y_{0}}{2}, \frac{x+y_{0}}{2}, 0\right)
\end{aligned}
$$

For all $x \in A$ and some $y_{0} \in A$ for which $x$ and $y_{0}$ satisfy the condition (c) with $\lambda=1$.

Proof: We define

$$
\mathcal{E}=\left\{f: \mathcal{A} \rightarrow \mathcal{B}, \sup _{\gamma(x)=\gamma(y)} \frac{\left\|f(x)-f_{1}(x)\right\|}{\psi(x, y)}<\infty\right\} .
$$

Define $d: \mathcal{E} \times \mathcal{E} \rightarrow[0, \infty]$ by

$$
d(f, g)=\sup _{\gamma(x)=\gamma(y)} \frac{\|f(x)-g(x)\|}{\psi(x, y)} .
$$

It is easy to see that $(\mathcal{E}, d)$ is a complete metric space. Let us consider the linear mapping $\Lambda: \mathcal{E} \rightarrow \mathcal{E}$ defined by

$$
\Lambda f(x)=\frac{1}{2^{\ell}} f\left(2^{\ell} x\right)
$$

For all $x \in A$. Let $f, g \in \mathcal{E}$ and let $C \in[0, \infty)$ be an arbitrary constant with $d(f, g)<C$. From the definition of $d$, we have 


$$
\frac{\|f(x)-g(x)\|}{\psi(x, y)} \leq C^{\prime}
$$

For all $x, y \in A$ with $\gamma(x)=\gamma(y)$. By the assumption and the last inequality, we get

$\frac{\|\Lambda f(x)-\Lambda g(x)\|}{\psi(x, y)}=\left\|f\left(2^{\ell} x\right)-g\left(2^{\ell} x\right)\right\| 2^{\ell} \psi(x, y) \leq \frac{L\left\|f\left(2^{\ell} x\right)-g\left(2^{\ell} x\right)\right\|}{\psi\left(2^{\ell} x, 2^{\ell} y\right)} \leq L C^{\prime}$

For all $x, y \in A$ with $\gamma(x)=\gamma(y)$ and therefore

$d(\Lambda f, \Lambda g) \leq L d(f, g)$.

So, $\Lambda$ is a strictly contractive mapping with the Lipschitz constant $L$.

From (3.1) we have

$$
\begin{aligned}
& \left\|f_{1}(2 x)-f_{2}(x)-f_{3}(x)\right\| \leq \quad(x, x, 0), \\
& \left\|f_{2}(x)+f_{3}(-x)\right\| \leq \varphi(x,-x, 0)
\end{aligned}
$$

For all $x \in A$

Let $x \in A$. By $\left(\mathrm{c}_{3}\right)$ for $\lambda=1$, there exists $y_{0} \in A$ such that $\gamma\left(x+y_{0}\right)=\gamma$ $\left(x-y_{0}\right)$ and $\gamma(2 x)=\gamma\left(2 y_{0}\right)$.

Hence

$$
\begin{aligned}
& \left\|f_{1}(2 x)-f_{2}\left(x+y_{0}\right)-f_{3}\left(x-y_{0}\right)\right\| \leq \varphi\left(x+y_{0}, x-y_{0}, 0\right), \\
& \left\|f_{1}(2 x)-f_{2}\left(x-y_{0}\right)-f_{3}\left(x+y_{0}\right)\right\| \leq \varphi\left(x-y_{0}, x+y_{0}, 0\right), \\
& \left\|f_{1}\left(2\left(x+y_{0}\right)\right)-f_{2}\left(2 y_{0}\right)-f_{3}(2 x)\right\| \leq \varphi\left(2 y_{0}, 2 x, 0\right) .
\end{aligned}
$$

Also, by the evenness of $\gamma$ we have

$$
\left\|f_{1}\left(2\left(x-y_{0}\right)\right)-f_{2}(2 x)-f_{3}\left(-2 y_{0}\right)\right\| \leq \varphi\left(2 x,-2 y_{0}, 0\right) .
$$

Substituting both $\mathrm{x}, \mathrm{y}$ in (3.1) by $x+y_{0}$ and $x-y_{0}$, we have

$$
\begin{aligned}
& \left\|f_{1}\left(2\left(x+y_{0}\right)\right)-f_{2}\left(x+y_{0}\right)-f_{3}\left(x+y_{0}\right)\right\| \leq \varphi\left(x+y_{0}, x+y_{0}, 0\right), \\
& \left\|f_{1}\left(2\left(x-y_{0}\right)\right)-f_{2}\left(x-y_{0}\right)-f_{3}\left(x-y_{0}\right)\right\| \leq \varphi\left(x-y_{0}, x-y_{0}, 0\right) .
\end{aligned}
$$

It follows from the inequalities (3.5) - (3.12) and the triangle inequality that

$$
\begin{aligned}
& \left\|f_{1}(4 x)-2 f_{1}(2 x)\right\| \leq\left\|f_{1}(4 x)-f_{2}(2 x)-f_{3}(2 x)\right\| \\
& +\left\|-f_{1}\left(2\left(x+y_{0}\right)\right)+f_{2}\left(2 y_{0}\right)+f_{3}(2 x)\right\| \\
& +\left\|-f_{1}\left(2\left(x-y_{0}\right)\right)+f_{2}(2 x)+f_{3}\left(-2 y_{0}\right)\right\| \\
& +\left\|-f_{2}\left(2 y_{0}\right)-f_{3}\left(-2 y_{0}\right)\right\| \\
& +\left\|f_{1}\left(2\left(x+y_{0}\right)\right)-f_{2}\left(x+y_{0}\right)-f_{3}\left(x+y_{0}\right)\right\| \\
& +\left\|f_{1}\left(2\left(x-y_{0}\right)\right)-f_{2}\left(x-y_{0}\right)-f_{3}\left(x-y_{0}\right)\right\| \\
& +\left\|-f_{1}(2 x)+f_{2}\left(x+y_{0}\right)+f_{3}\left(x-y_{0}\right)\right\| \\
& +\left\|-f_{1}(2 x)+f_{2}\left(x-y_{0}\right)+f_{3}\left(x+y_{0}\right)\right\| \\
& \leq \varphi(2 x, 2 x, 0)+\varphi\left(2 y_{0}, 2 x, 0\right) \\
& +\varphi\left(2 x,-2 y_{0}, 0\right)+\varphi\left(2 y_{0},-2 y_{0}, 0\right) \\
& +\varphi\left(x+y_{0}, x+y_{0}, 0\right)+\varphi\left(x-y_{0}, x-y_{0}, 0\right)
\end{aligned}
$$

$$
\begin{aligned}
& +\varphi\left(x+y_{0}, x-y_{0}, 0\right)+\varphi\left(x-y_{0}, x+y_{0}, 0\right) \\
& =\psi\left(2 x, 2 y_{0}\right) .
\end{aligned}
$$

If we replace $x$ and $y_{0}$ in (3.13) with $\frac{x}{2}$ and $\frac{y_{0}}{2}$, respectively, and divide by 2 the resulting inequality, then we have

$$
\left\|f_{1}(x)-\frac{1}{2} f_{1}(2 x)\right\| \leq \frac{1}{2} \psi\left(x, y_{0}\right) .
$$

If we replace $x$ and $y_{0}$ in (3.13) with $\frac{x}{4}$ and $\frac{y_{0}}{4}$, respectively, and using 3-expansivity of $\varphi$, then

$$
\left\|f_{1}(x)-2 f_{1}\left(\frac{x}{2}\right)\right\| \leq \psi\left(\frac{x}{2}, \frac{y_{0}}{2}\right) \leq \frac{L^{2}}{4} \psi\left(x, y_{0}\right) .
$$

We can reduce (3.14) and (3.15) to

$$
\left\|f_{1}(x)-\frac{1}{2^{\ell}} f_{1}\left(2^{\ell} x\right)\right\| \leq \frac{L^{1-\ell}}{2} \psi\left(x, y_{0}\right)
$$

and then

$$
\frac{\left\|f_{1}(x)-\frac{1}{2^{\ell}} f_{1}\left(2^{\ell} x\right)\right\|}{\psi\left(x, y_{0}\right)} \leq \frac{L^{1-\ell}}{2},
$$

That is, $d\left(f_{1}, \Lambda f_{1}\right) \leq \frac{L^{1-\ell}}{2}$. By Theorem 2.5, there exists a unique mapping $H \in \mathcal{E}$ which is the fixed point of

$\Lambda$ and satisfies

$H(x)=\lim _{n \rightarrow \infty} \frac{f_{1}\left(2^{n \ell} x\right)}{2^{n \ell}}$.

Also

$d\left(f_{1}, H\right) \leq \frac{1}{1-L} d\left(f_{1}, \Lambda f_{1}\right) \leq \frac{L^{1-\ell}}{2(1-L)}$

This yield

$\left\|f_{1}(x)-H(x)\right\| \leq \frac{L^{1-\ell}}{2(1-L)} \psi\left(x, y_{0}\right)$.

Let $x \in A$ and $y_{0} \in A$ be the element for which $x$ and $y_{0}$ satisfy the condition $\left(c_{3}\right)$ with $\lambda=1$. Utilizing the triangle inequality, the oddness of $f_{3}$ and the inequalities (3.5) and (3.6), we infer that

$$
\begin{aligned}
& \left\|2 f_{2}(x)-f_{1}(2 x)\right\| \leq\left\|-f_{1}(2 x)+f_{2}(x)+f_{3}(x)\right\| \\
& +\left\|f_{2}(x)+f_{3}(-x)\right\| \\
& \leq \varphi(x, x, 0)+\varphi(x,-x, 0), \\
& \left\|2 f_{3}(x)-f_{1}(2 x)\right\| \leq\left\|-f_{1}(2 x)+f_{2}(x)+f_{3}(x)\right\| \\
& +\left\|-f_{2}(x)-f_{3}(-x)\right\| \\
& \leq \varphi(x, x, 0)+\varphi(x,-x, 0) .
\end{aligned}
$$

Combining (3.17) and (3.18) with (3.16), we get

$$
\begin{aligned}
& \left\|2 f_{2}(x)-H(2 x)\right\| \leq\left\|f_{1}(2 x)-H(2 x)\right\|+\left\|2 f_{2}(x)-f_{1}(2 x)\right\|, \\
& \left\|2 f_{3}(x)-H(2 x)\right\| \leq\left\|f_{1}(2 x)-H(2 x)\right\|+\left\|2 f_{3}(x)-f_{1}(2 x)\right\| .
\end{aligned}
$$

Using the above inequalities and the fact that $H(2 x)=2 H(x)$, we obtain 


$$
\begin{gathered}
\left\|f_{2}(x)-H(x)\right\| \leq \frac{L^{3-\ell}}{1-L} \psi\left(x, y_{0}\right)+\frac{1}{2}(\varphi(x, x, 0)+\varphi(x,-x, 0)), \\
\left\|f_{3}(x)-H(x)\right\| \leq \frac{L^{3-\ell}}{1-L} \psi\left(x, y_{0}\right)+\frac{1}{2}(\varphi(x, x, 0)+\varphi(x,-x, 0)) .
\end{gathered}
$$

From the inequalities (3.17) and (3.18), we get

$$
\begin{aligned}
& \left\|2^{-n \ell} f_{2}\left(2^{n \ell} x\right)-2^{-(n+1) \ell} f_{1}\left(2^{(n+1) \ell} x\right)\right\| \\
& \leq 2^{-(n+1) \ell}\left(\varphi\left(2^{n \ell} x, 2^{n \ell} x, 0\right)+\varphi\left(2^{n \ell} x,-2^{n \ell} x, 0\right)\right), \\
& \left\|2^{-n \ell} f_{3}\left(2^{n \ell} x\right)-2^{-(n+1) \ell} f_{1}\left(2^{(n+1) \ell} x\right)\right\| \\
& \leq 2^{-(n+1) \ell}\left(\varphi\left(2^{n \ell} x, 2^{n \ell} x, 0\right)+\varphi\left(2^{n \ell} x,-2^{n \ell} x, 0\right)\right)
\end{aligned}
$$

For all $n \in \mathbb{N}$, whence

$$
H(x)=\lim _{n \rightarrow \infty} \frac{f_{1}\left(2^{n \ell} x\right)}{2^{n \ell}}=\lim _{n \rightarrow \infty} \frac{f_{2}\left(2^{n \ell} x\right)}{2^{n \ell}}=\lim _{n \rightarrow \infty} \frac{f_{3}\left(2^{n \ell} x\right)}{2^{n \ell}} .
$$

Let $x, y \in A$ with $\gamma(x)=\gamma(y)$. By the assumption, $\gamma\left(2^{\ell} x\right)=\gamma\left(2^{\ell} y\right)$ and then $\gamma\left(2^{n \ell} x\right)=\gamma\left(2^{n \ell} y\right)$ for all $n \in \mathbb{N}$. By 3-contractivity of $\varphi$ we infer that

$$
\left\|2^{-n} f_{1}\left(2^{n}(x+y)\right)-2^{-n} f_{2}\left(2^{n} x\right)-2^{-n} f_{3}\left(2^{n} y\right)\right\| \leq 2^{-n} \varphi\left(2^{n} x, 2^{n} y, 0\right) \leq\left(2 L^{2}\right)^{n} \varphi(x, y, 0) .
$$

And by 3-expansivity of $\varphi$ we conclude that

$\left\|2^{n} f_{1}\left(2^{-n}(x+y)\right)-2^{n} f_{2}\left(2^{-n} x\right)-2^{n} f_{3}\left(2^{-n} y\right)\right\| \leq 2^{n} \varphi\left(2^{-n} x, 2^{-n} y, 0\right) \leq\left(\frac{L^{2}}{2}\right)^{n} \varphi(x, y, 0)$.

Since $L<\frac{1}{\sqrt{2}}$, from $n \rightarrow \infty$ in the above inequalities, one proves by (3.21) that $H(x+y)-H(x)-H(y)=0$. Hence $H$ is conditionally additive.

In addition, it is clear from (3.1) that the following inequality

$$
\begin{aligned}
& \|H(t z)-t H(z)\|=\lim _{n \rightarrow \infty} \frac{1}{2^{n \ell}}\left\|f_{1}\left(2^{n \ell} t z\right)-t f_{1}\left(2^{n \ell} z\right)\right\| \\
& \leq \lim _{n \rightarrow \infty} \frac{1}{2^{n \ell}} \varphi\left(0,0,2^{n \ell} z\right) \\
& \leq \lim _{n \rightarrow \infty} L^{n} \varphi(0,0, z)=0
\end{aligned}
$$

Holds for all $x \in A$ and $t \in \mathbb{T}_{1 / n_{0}}^{1}$. By Lemma 3.1, $H$ is $\mathbb{C}$-linear.

We claim that the mapping $H$ satisfies the functional equation (2.1). Define $r: \mathcal{A}^{2} \rightarrow \mathcal{B}$ by $r(x, y)=f_{1}([x, y])-\left[f_{2}(x), f_{3}(y)\right]$ for all $x, y$ $\in A$ with $\gamma(x)=\gamma(y)$. From (3.2) it follows that

$$
\lim _{n \rightarrow \infty} \frac{r\left(2^{n \ell} x, y\right)}{2^{n \ell}}=0 .
$$

Making use of (3.21) and (3.22), we get

$$
\begin{aligned}
H([x, y]) & =\lim _{n \rightarrow \infty} \frac{f_{1}\left(2^{n \ell}[x, y]\right)}{2^{n \ell}}=\lim _{n \rightarrow \infty} \frac{f_{1}\left(\left[2^{n \ell} x, y\right]\right)}{2^{n \ell}} \\
& =\lim _{n \rightarrow \infty} \frac{\left[f_{2}\left(2^{n \ell} x\right), f_{3}(y)\right]+r\left(2^{n \ell} x, y\right)}{2^{n \ell}} \\
& =\lim _{n \rightarrow \infty}\left(\left[\frac{f_{2}\left(2^{n \ell} x\right)}{2^{n \ell}}, f_{3}(y)\right]+\frac{r\left(2^{n \ell} x, y\right)}{2^{n \ell}}\right) \\
& =\left[H(x), f_{3}(y)\right]
\end{aligned}
$$

For all $x, y \in A$ with $\gamma(x)=\gamma(y)$.
Now, letting $x, y \in A$ with $\gamma(x)=\gamma(y)$ and $n \in \mathbb{N}$, by (3.23) and conditional additivity of $H$ one obtains

$$
\begin{aligned}
{\left[H(x), f_{3}\left(2^{n \ell} y\right)\right] } & =H\left(\left[x, 2^{n \ell} y\right]\right)=H\left(\left[2^{n \ell} x, y\right]\right) \\
= & {\left[H\left(2^{n \ell} x\right), f_{3}(y)\right]=2^{n \ell}\left[H(x), f_{3}(y)\right] }
\end{aligned}
$$

This yield

$$
\left[H(x), \frac{f_{3}\left(2^{n \ell} y\right)}{2^{n \ell}}\right]=\left[H(x), f_{3}(y)\right] .
$$

The comparison of the above equality with (3.23) shows that

$$
H([x, y])=\left[H(x), \frac{f_{3}\left(2^{n \ell} y\right)}{2^{n \ell}}\right] .
$$

For all $x, y \in A$ with $\gamma(x)=\gamma(y)$ and $n \in \mathbb{N}$. Taking the limit as $n \rightarrow$ $\infty$, we conclude that $\mathrm{H}([x, y])=[\mathrm{H}(x), \mathrm{H}(y)]$. This completes the proof of Theorem 3.2.

In particular, given $\varphi(x, y, z)=\varepsilon\left(\|x\|^{p}+\|y\|^{p}+\|z\|^{p}\right)$ and $\phi(x, y)=\theta\left(\|x\|^{p}+\|y\|^{p}\right)$ for $\varepsilon, \theta \geq 0$ and some real numbers $p$ in the main theorem, one gets the following corollary.

Corollary 3.3: Let $f_{i}: \mathcal{A} \rightarrow \mathcal{B}(i=1,2,3)$ be mappings satisfying $\left\|f_{1}(x+y)-f_{2}(x)-f_{3}(y)+f_{i}(t z)-t f_{i}(z)\right\| \leq \varepsilon\left(\|x\|^{p}+\|y\|^{p}+\|z\|^{p}\right)$,

$$
\left\|f_{1}([x, y])-\left[f_{2}(x), f_{3}(y)\right]\right\| \leq \theta\left(\|x\|^{p}+\|y\|^{p}\right)
$$

For all $t \in \mathbb{T}_{1 / n_{0}}^{1}$ and $x, y, z \in A$ with $\|x\|=\|y\|, \varepsilon, \theta \geq 0$ and real numbers $p$ such that $p<1$ for $\ell=1$ and $p>1$ for $\ell=-1$. If $f_{3}$ is an odd mapping and $f_{i}(0)=0(i=1,2,3)$, then there exists a unique conditional homomorphism $H: \mathcal{A} \rightarrow \mathcal{B}$ such that

$$
\begin{aligned}
& \left\|f_{1}(x)-H(x)\right\| \\
& \quad \leq \varepsilon \frac{2^{\ell(1-\ell)(p-1)}}{2\left(1-2^{\ell(p-1)}\right)}\left(\left\|\frac{x+y_{0}}{2}\right\|^{p}+\left\|\frac{x-y_{0}}{2}\right\|^{p}+\|x\|^{p}+\left\|y_{0}\right\|^{p}\right) \\
& \left\|f_{2}(x)-H(x)\right\| \\
& \quad \leq \varepsilon \frac{2^{\ell(3-\ell)(p-1)}}{1-2^{\ell(p-1)}}\left(\left\|\frac{x+y_{0}}{2}\right\|^{p}+\left\|\frac{x-y_{0}}{2}\right\|^{p}+\|x\|^{p}+\left\|y_{0}\right\|^{p}\right)+2\|x\|^{p},
\end{aligned}
$$

$\left\|f_{3}(x)-H(x)\right\|$

$$
\leq \varepsilon \frac{2^{\ell(3-\ell)(p-1)}}{1-2^{\ell(p-1)}}\left(\left\|\frac{x+y_{0}}{2}\right\|^{p}+\left\|\frac{x-y_{0}}{2}\right\|^{p}+\|x\|^{p}+\left\|y_{0}\right\|^{p}\right)
$$$$
+2\|x\|^{p}
$$

For all $x \in A$ and some $y_{0} \in A$ for which $x$ and $y_{0}$ satisfy the condition (c) with $\lambda=1$.

Proof: Let $\gamma:=\|\cdot\|$ (cf. Example 2.2). Define $\varphi$ and $\phi$ as above and apply Theorem 3.2 with $L=2^{\ell(p-1)}$.

In the next theorem, we prove the Hyers-Ulam stability problem for conditional Jordan homomorphisms.

Theorem 3.4: Suppose that $f_{i}: \mathcal{A} \rightarrow \mathcal{B} \quad(i=1,2,3)$ are mappings satisfying the functional inequalities

$$
\begin{aligned}
& \left\|f_{1}(x+y)-f_{2}(x)-f_{3}(y)+f_{i}(t z)-t f_{i}(z)\right\| \leq \varphi(x, y, z), \\
& \left\|f_{1}([x, y]+[y, x])-\left[f_{2}(x), f_{3}(y)\right]-\left[f_{2}(y) f_{3}(x)\right]\right\| \leq \phi(x, y)
\end{aligned}
$$

For all $t \in \mathbb{T}_{1 / n_{0}}^{1}$ and $x, y, z \in A$ with $\gamma(x)=\gamma(y)$. If $f_{3}$ is an odd mapping and $f_{i}(0)=0(i=1,2,3)$, then there exists a unique conditional 
Citation: Eshaghi M, Abbaszadeh S, Sen MD (2015) On The Stability of Conditional Homomorphisms in Lie C*-algebras. J Generalized Lie Theory Appl 9: 220. doi:10.4172/1736-4337.1000220

Page 5 of 5

Jordan homomorphism $H: \mathcal{A} \rightarrow \mathcal{B}$ satisfying (3.3).

Proof: Applying a similar argument to the corresponding part of Theorem 3.2, we can conclude that there exists a unique conditional $\mathbb{C}$ -linear mapping $H: \mathcal{A} \rightarrow \mathcal{B}$ satisfying (3.3). Moreover,

$$
H(x)=\lim _{n \rightarrow \infty} \frac{f_{1}\left(2^{n \ell} x\right)}{2^{n \ell}}=\lim _{n \rightarrow \infty} \frac{f_{2}\left(2^{n \ell} x\right)}{2^{n \ell}}=\lim _{n \rightarrow \infty} \frac{f_{3}\left(2^{n \ell} x\right)}{2^{n \ell}}
$$

For all $\mathrm{x} \in \mathrm{A}$. We will show that $\mathrm{H}$ satisfies the functional equation (2.2). Define $r: \mathcal{A}^{2} \rightarrow \mathcal{B}$ by $r(x, y)=f_{1}([x, y]+[y, x])-\left[f_{2}(x), f_{3}(y)\right]-\left[f_{2}(y), f_{3}(x)\right]$ for all $x, y \in A$ with $\gamma(x)=\gamma(y)$. It follows from (3.26) that

$$
\lim _{n \rightarrow \infty} \frac{r\left(2^{n \ell} x, y\right)}{2^{n \ell}}=0 .
$$

By (3.27) and (3.28), we obtain

$$
\begin{aligned}
H([x, y]+[y, x]) & =\lim _{n \rightarrow \infty} \frac{f_{1}\left(2^{n \ell}([x, y]+[y, x])\right)}{2^{n \ell}} \\
& =\lim _{n \rightarrow \infty} \frac{f_{1}\left(\left[2^{n \ell} x, y\right]+\left[y, 2^{n \ell} x\right]\right)}{2^{n \ell}} \\
& =\lim _{n \rightarrow \infty} \frac{\left[f_{2}\left(2^{n \ell} x\right), f_{3}(y)\right]+\left[f_{2}(y), f_{3}\left(2^{n \ell} x\right)\right]+r\left(2^{n \ell} x, y\right)}{2^{n \ell}} \\
& =\left[H(x), f_{3}(y)\right]+\left[f_{2}(y), H(x)\right]
\end{aligned}
$$

For all $x, y \in A$ with $\gamma(x)=\gamma(y)$.

Now let $x, y \in A$ with $\gamma(x)=\gamma(y)$ and $n \in \mathbb{N}$ be fixed. By (3.29) and conditional additivity of $H$, it can be shown that

$$
\begin{aligned}
{\left[H(x), f_{3}\left(2^{n \ell} y\right)\right]+\left[f_{2}\left(2^{n \ell} y\right), H(x)\right] } & =H\left(\left[x, 2^{n \ell} y\right]+\left[2^{n \ell} y, x\right]\right) \\
& =H\left(\left[2^{n \ell} x, y\right]+\left[y, 2^{n \ell} x\right]\right) \\
& =\left[H\left(2^{n \ell} x\right), f_{3}(y)\right]+\left[f_{2}(y), H\left(2^{n \ell} x\right)\right] \\
& =2^{n \ell}\left[H(x), f_{3}(y)\right]+2^{n \ell}\left[f_{2}(y), H(x)\right]
\end{aligned}
$$

and then

$$
\left[H(x), \frac{f_{3}\left(2^{n \ell} y\right)}{2^{n \ell}}\right]+\left[\frac{f_{2}\left(2^{n \ell} y\right)}{2^{n \ell}}, H(x)\right]=H([x, y]+[y, x]) .
$$

Sending $n$ to infinity, we obtain

$$
H([x, y]+[y, x])=[H(x), H(y)]+[H(y), H(x)] .
$$

This completes the proof of the theorem.

Corollary 3.5: Let $f_{i}: \mathcal{A} \rightarrow \mathcal{B}(i=1,2,3)$ be mappings satisfying

$$
\begin{aligned}
& \left\|f_{1}(x+y)-f_{2}(x)-f_{3}(y)+f_{i}(t z)-t f_{i}(z)\right\| \leq \varepsilon\left(\|x\|^{p}+\|y\|^{p}+\|z\|^{p}\right), \\
& \left\|f_{1}([x, y]+[y, x])-\left[f_{2}(x), f_{3}(y)\right]-\left[f_{2}(y) f_{3}(x)\right]\right\| \leq \theta\left(\|x\|^{p}+\|y\|^{p}\right)
\end{aligned}
$$

For all $t \in \mathbb{T}_{1 / n_{0}}^{1}$ and $x, y, z \in A$ with $\|x\|=\|y\|, \varepsilon, \theta \geq 0$ and real numbers $p$ such that $p<1$ for $\ell=1$ and $p>1$ for $\ell=-1$. If $f_{3}$ is an odd mapping and $f_{i}(0)=0(i=1,2,3)$, then there exists a unique conditional Jordan homomorphism $H: \mathcal{A} \rightarrow \mathcal{B}$ satisfying (3.25).

Proof: $\quad$ Define $\gamma:=\|\cdot\|, \quad \varphi(x, y, z)=\varepsilon\left(\|x\|^{p}+\|y\|^{p}+\|z\|^{p}\right) \quad$ and $\phi(x, y)=\theta\left(\|x\|^{p}+\|y\|^{p}\right)$ apply Theorem 3.4 with $\mathrm{L}=2^{\ell(p-1)}$.

\section{Acknowledgement}

The authors are very grateful to the Spanish Government for its support of this research through Grant DPI2012-30651.

\section{References}

1. Morton TM, Neamtu M (2002) Error bounds for solving pseudo differential equations on spheres by collocation with zonal kernels. J Approx Theory 114 242-268.

2. LeGia QT, Mhaskar HN (2006) Polynomial operators and local approximation of solution of pseudo-differential equations on the sphere. Numer Math 103: 299-322.

3. Tran T, LeGia QT, Sloan LH, Stephan EP (2010) Pre conditioners for pseudo differential equations on the sphere with radial basis functions. Numer Math 115: 141-163.

4. Ulam SM (1960) A Collection of Mathematical Problems. Interscience Publications, New York.

5. Hyers DH (1941) On the stability of the linear functional equation. Proc Nat Acad Sci USA 27: 222-224.

6. Rassias TM (1978) On the stability of the linear mapping in Banach spaces Proc Am Math Soc 72: 297-300.

7. Rassias JM (1982) On Approximation of Approximately Linear Mappings by Linear Mappings. J Func Anal 46: 126-130.

8. Rassias TM (1991) On a modified Hyers-Ulam sequence. J Math Anal App 158: 106-113.

9. Gavruta P (1994) A generalization of the Hyers-Ulam-Rassias stability of approximately additive mappings. J Math Anal Appl 184: 431-436.

10. Alsina C, Garcia-Roig JL (1994) On a conditional Cauchy equation on rhombuses. In: Functional Analysis, Approximation Theory and Numerical Analysis. World Sci Publishing, River Edge, NJ.

11. Sikorska J (2008) Generalized stability of the cauchy and jensen functional equations on spheres. J Math Anal Appl 345: 650-660.

12. Sikorska J (2009) on two conditional pexider functional equations and their stabilities. Nonlinear Anal 70: 2673-2684.

13. Sikorska J (2009) On a pexiderized conditional exponential functional equation. Acta Math Hungar 125: 287-299.

14. Brzdek J, Sikorska J (2010) A conditional exponential functional equation and its stability. Nonlinear Anal TMA 72: 2923-2934.

15. Brzdek J (2010) On the stability of a family of functional equations. Acta Math Hungar 128: 139-149.

16. Fechner W, Sikorska J (2012) On a separation for the Cauchy equation on spheres. Nonlinear Anal TMA 75: 6306-6311.

17. Chung JY (2012) Stability of a conditional Cauchy equation. Aequat Math 83 313-320.

18. Ger R, Sikorska J (1997) On the Cauchy equation on spheres. Ann Math Sil 11: 89-99.

19. Park C (2004) Lie *-homomorphisms between Lie C*-algebras and Lie*derivations on Lie C*-algebras. J Math Anal Appl 293: 419-434.

20. Park C (2005) Homomorphisms between Lie J C*-algebras and CauchyRassias stability of Lie J C*-algebra derivations. J Lie Theory 15: 393-414.

21. Gordji ME, Khodaei $H$ (2013) A fixed point technique for investigating the stability of $(\alpha, \beta, \gamma)$-derivations on Lie $C^{*}$-algebras. Nonlinear Anal 76: 52-57.

22. Eshaghi Gordji M, Najati A (2010) Approximately j*-homomorphisms: a fixed point approach. J Geom Phys 60: 809-814.

23. Eshaghi Gordji M (2013) Nearly involutions on banach algebras: a fixed point approach. J Math Phys 14: 117-124. 